\title{
Coupling Photosphere and Corona: Linear and Turbulent Regimes
}

\author{
Verdini A.*, Grappin R. ${ }^{\dagger}$ and Velli M.*, \\ *SIDC, Observatoire Royal de Belgique, Bruxelles, Belgium \\ ${ }^{\dagger}$ LUTH, Observatoire de Paris, France \\ ${ }^{* *}$ Dipartimento di Astronomia e Scienza dello Spazio, Firenze, Italy \\ $¥$ Jet Propulsion Laboratory, California Institute of Technology, Pasadena, CA 91109, USA
}

\begin{abstract}
In a recent work Grappin et al. [1] have shown that low- frequency movements can be transmitted from one footpoint to the other along a magnetic loop, thus mimicking a friction effect of the corona on the photosphere, and invalidating the line-tying approximation. We consider here successively the effect of high frequencies and turbulent damping on the process. We use a very simple atmospheric model which allows to study analytically the laminar case, and to study the turbulent case both using simple phenomenological arguments and a more sophisticated turbulence model [2]. We find that, except when turbulent damping is such that all turbulence is damped during loop traversal, coupling still occurs between distant footpoints, and moreover the coronal field induced by photospheric movements saturates at finite values.
\end{abstract}

Keywords: MHD, Coornal Loops, Waves; Turbulence

PACS: $96.60 . p f, 96.60 . \mathrm{Mz}, 96.60 . \mathrm{Na}$

In large simulations the line-tying approximation is usually adopted for the coronal boundary. According to this approximation, strong density gradients cause the reflection of any coronal disturbance. Hence, the photospheric velocity field can be prescribed since the reaction of the coronal dynamics is completely neglected. Applying a velocity field $u_{0}$ at the base of a loop of length $L$ and mean magnetic field $B_{0}$, keeping the other foot anchored in the photosphere leads to an infinite accumulation of magnetic energy (driven by the velocity shear between the loop footpoints). To test whether the stratification really leads to line-tying, Grappin et al. [1] have studied the transmission of a signal from one footpoint to the other including a simple atmospheric model in a 1.5D MHD simulation. Footpoints are free to move, i.e. boundaries are transparent to waves, and a kick is given to left footpoint, injecting an Alfvén waves from the left boundary. At the beginning, the magnetic energy grows steadily according to the line-tied assumption, $b \approx B_{0} u_{0} t / L$ (transient acceleration of the left footpoint). On longer times $\tau_{r e l}=L / V_{a}^{p h}$ ( $V_{a}^{p h}$ is the Alfvén speed at the photosphere), the leakage of Alfvén waves through the transition region (T.R.) accelerates (decelerates) the right (left) footpoint: the system relaxes to a state in which both footpoints have the same speed.

\section{SIMPLIFIED MODEL ATMOSPHERE}

We adopt a rough representation of the loop atmosphere modeling the transition region as a discontinuity in the
Alfvén speed, separating two uniform Alfvén speed chromosphere and corona (hereafter subscript and/or superscript $c, p h$ indicate quantities evaluated at the corona and at the photosphere, not distinguished from the chromosphere in the model). This allows to easily consider the effect of finite frequency, wave reflection, and wave damping while retaining the physical process responsible for the relaxation.

The small parameter $\varepsilon=V_{a}^{p h} / V_{a}^{c}$ quantifies the jump at the T.R. and hence reflection. Continuity of magnetic and velocity field fluctuation, $\delta b, \delta u$, at the two T.R.s yields four jump conditions for the Elsässer variables $u^{ \pm}=\delta u \mp \delta b / \sqrt{4 \pi \rho}$ :

$$
\begin{gathered}
u_{1}^{+}+u_{1}^{-}=u_{L}^{+}+u_{L}^{-}, \quad u_{1}^{+}-u_{1}^{-}=\left(u_{L}^{+}-u_{L}^{-}\right) / \varepsilon \\
u_{R}^{+}+u_{R}^{-}=u_{3}^{+}, \quad u_{R}^{+}-u_{R}^{-}=u_{3}^{+} / \varepsilon, \quad\left(u_{3}^{-}=0\right),
\end{gathered}
$$

here labeled $u_{1, L, R, 3}^{ \pm}$. Superscripts \pm indicate the direction of propagation (rightward and leftward respectively), while $1, L, R, 3$ specifies the position with respect to the T.R. where they are evaluated: left chromosphere, left corona, right corona, right chromosphere respectively. We further use the notation $p h, 1$ and $p h, 3$ for evaluations at the left and right footpoints (see Fig. 1).

We chose solutions of the form $u \propto \exp [-i \omega t-t / \tau]$ in which $\omega$ is the wave frequency and $\tau$ is a damping timescale. A wave of amplitude $u_{p h, 1}^{+}=u_{1}^{0}$ is injected at the left footpoint so that $u_{1}^{+}=u_{1}^{0} \exp \left[-i \omega t_{p h}\right]$. Time is counted in coronal crossing time $t=n t_{c}=n L_{c} / V_{a}^{c}, n$ indicating the number of reflections, so that wave propagation and damping in the corona (we exclude damping in the chromosphere for reasons that will be clear 


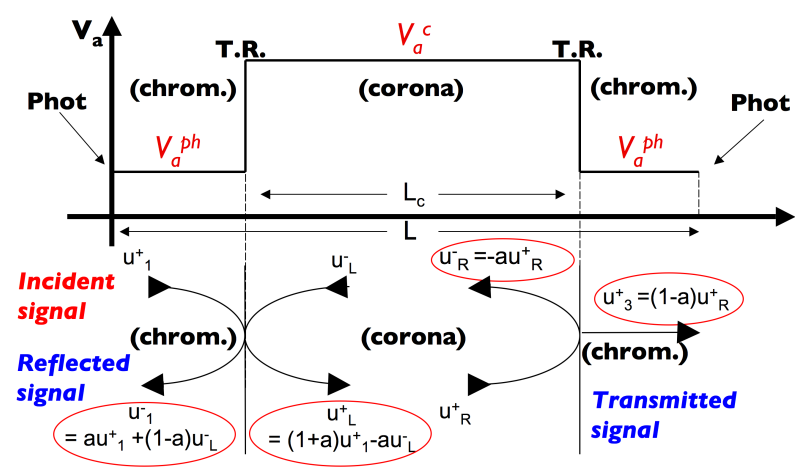

FIGURE 1. Modeled loop atmosphere (top) and jump conditions (red circles) at the two T.R.s (bottom) for the rightward and leftward propagating waves $\left(u^{ \pm}\right.$respectively).

after) are accounted by the factors $\xi=\exp \left[-i \omega t_{c}\right]$ and $\beta=\exp \left[-t_{c} / \tau\right]$ :

$$
\left.u_{R}^{+}\right|_{n+1}=\left.\xi \beta u_{L}^{+}\right|_{n},\left.\quad u_{L}^{-}\right|_{n+1}=\left.\xi \beta u_{R}^{-}\right|_{n} .
$$

Combining Eqs. 1-3 during a cycle of reflection and propagation in the corona yields a recurrence formula, $a=(1-\varepsilon) /(1+\varepsilon)$,

$$
\left.u_{L}^{+}\right|_{n}=(1+a) u_{1}^{+}\left|n+a^{2} \beta^{2} \xi^{2} u_{L}^{+}\right|_{n-2}
$$

(and a similar expression for $u_{L}^{-}$) that can be used to evaluate for each variable $u_{i}^{ \pm}$: a) its asymptotic value, setting $n=n-2 ;$ b) its temporal evolution, expliciting $\left.u\right|_{n-2}=f\left(\left.u_{1}^{+}\right|_{n-4},\left.u\right|_{n-4}\right)$ and so on.

Here we consider only two special cases: a) Finite frequency fluctuations $(\xi \neq 1)$ with no damping; b) Zero frequency fluctuation with damping $(\beta \neq 1)$.

\section{NO DAMPING, $\omega \neq 0$}

With $\beta=1$, the asymptotic solutions for the reflected and transmitted wave can be written as

$$
u_{1}^{-}=a \frac{1-\xi^{2}}{\left(1-a^{2} \xi^{2}\right)} u_{1}^{+}, \quad u_{3}^{+}=\frac{\left(1-a^{2}\right) \xi}{\left(1-a^{2} \xi^{2}\right)} u_{1}^{+},
$$

while in the corona one finds

$$
\begin{array}{r}
u_{L}^{+}=\frac{(1+a) \xi}{\left(1-a^{2} \xi^{2}\right)} u_{1}^{+}, \quad u_{R}^{+}=\xi u_{L}^{+} \\
u_{L}^{-}=-a \frac{(1+a) \xi^{2}}{\left(1-a^{2} \xi^{2}\right)} u_{1}^{+}, \quad u_{R}^{-}=-a u_{R}^{+}
\end{array}
$$

For injected waves of vanishing frequency and very low coronal density (small $\varepsilon$ ) one finds immediately from Eqs. 5-7 that $u_{L}^{+} \approx 1 /(1-a) u_{1}^{+} \approx 1 / \varepsilon u_{1}^{+} \gg u_{1}^{+}$and $u_{3}^{+}=$ $(1-a) u_{R}^{+} \approx 2 \varepsilon u_{R}^{+}$. Hence full transmission $\left(u_{1}^{+} \approx u_{3}^{+}\right)$

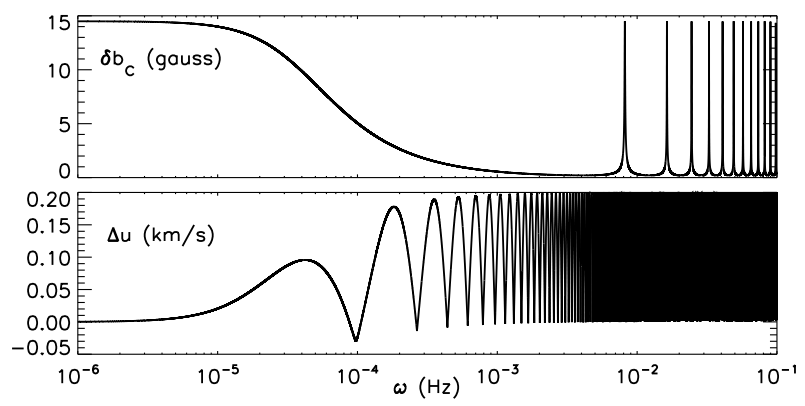

FIGURE 2. Asymptotic shear between footpoints $\Delta u$ (bottom) and coronal magnetic field (top) as a function of frequency.

implies very large coronal amplitudes $u_{c}^{ \pm} \approx 1 / \varepsilon$ (larger than the WKB prediction $u_{W K B} \approx 1 / \sqrt{\varepsilon}$ ) and also $u_{c}^{+} \approx$ $-u_{c}^{-}$. The latter finally implies $\delta b / \sqrt{4 \pi \rho} \gg \delta u$, that is magnetic energy much larger than kinetic energy in the corona. The asymptotic solution is actually a uniform $\delta u$ and $\delta b$ along the loop, while line-tying would have given a linear profile for $\delta u$ (from $u_{1}^{0}$ to 0 ) and $\delta b$ that goes to $\infty$ (never ending shear) $^{1}$.

The asymptotic velocity shear between the right and left footpoints $\Delta u=\left|\delta u_{p h, 1}\right|-\left|\delta u_{p h, 3}\right|$ and the coronal magnetic field $\delta b_{c}$ for finite frequency fluctuations are shown in Fig. 2 as obtained from Eqs. 5-7 assuming a $10 \mathrm{Mm}$ loop, $L_{p h}=2 \mathrm{Mm}, V_{a}^{p h}=0.7 \mathrm{~km} / \mathrm{s}$, and $\varepsilon=1 / 70 \ll 1$. $\delta b_{c}$ ranges from the photospheric value, $\delta b_{p h}=15$ Gauss, at zero frequency to zero at high frequencies. Because of the almost perfect reflection at the two T.R.s the coronal part of the loop behaves as a resonant cavity (compare the poster [3] in the same session and [4]), producing peaks at the fundamental frequency $V_{a}^{c} / L_{c}$ and its harmonics. On the other hand the asymptotic shear (Fig. 2, bottom panel) has well defined peaks well below such frequency that are caused by the phase shift between the injected and reflected waves at the left footpoint, i.e. a dependence of the form $\Delta u=g\left(\omega t_{p h}\right), t_{p h}=L_{p h} / V_{a}^{p h}$ being the chromospheric crossing time. Figure 3 shows the temporal evolution of $\Delta u$ for different frequencies (black lines) for the same $10 \mathrm{Mm}$ loop. The relaxation is independent of $\varepsilon$ (cf. the plots for $\omega=0 \mathrm{~Hz}$ obtained with $\varepsilon=1 / 70$ and $\varepsilon=0.4$ ), which allows to derive a compact formula for $\Delta u(t)$ by expliciting the dependence $u_{n-2}=f\left(u_{n-4}\right)=f\left(u_{n-6}\right) \ldots$ and taking the limit $\varepsilon \rightarrow 0$ :

$$
\begin{aligned}
& u_{1}^{-}=u_{1}^{+} a\left[1-\left(1-a^{2}\right) \xi^{2} \mathscr{H}(t)\right] \\
& u_{3}^{+}=u_{1}^{+}\left(1-a^{2}\right) \xi \mathscr{H}(t)
\end{aligned}
$$

\footnotetext{
1 the line-tying can be recovered taking the limit $\varepsilon \rightarrow 0$ before $n \rightarrow \infty$
} 


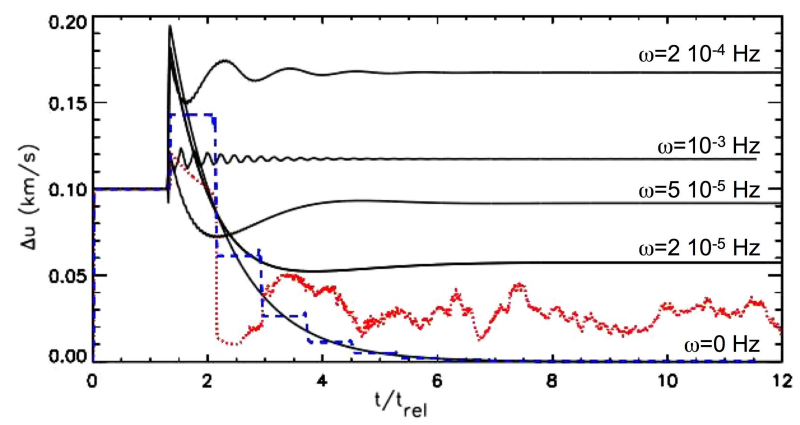

FIGURE 3. Velocity difference between footpoints $\Delta u$ as a function of time for different frequencies, for $\omega=0$ and $\varepsilon=0.4$ (blue-dashed), and for the weakly nonlinear case (red-dotted).

with; $\mathscr{H}(t)=\frac{1-\exp \left[-2 t / \tau_{\text {rel }}-i \omega t\right]}{1+i \omega \tau_{\text {rel }}}$

and $\tau_{\text {rel }}=L_{c} / V_{a}^{p h}$ being the relaxation timescale analogous $^{2}$ to that found by Grappin et al. [1], $\tau_{r e l}=L / V_{a}^{p h}$. The peak at $t \approx 2 t_{p h}$ corresponds to the initial acceleration of the left footpoint exerted by the first reflection at the T.R. (transient line-tying). The following relaxation is due to the deceleration of the left footpoint and the acceleration of the right footpoint. Oscillations in $\Delta u(t)$ for finite frequencies are caused by the phase shift between the injected and reflected waves at the left photosphere (the $i \omega t$ term).

\section{TURBULENT DAMPING, $\omega=0$}

We are now interested in understanding whether the above relaxation (for zero frequency) takes place in presence of a damping, that we chose to be caused by incompressible turbulence triggered by counter-propagating waves. We perform simulations in which the nonlinear MHD dynamics in planes perpendicular to the mean magnetic field is replaced by two-dimensional shell models for $\delta u$ and $\delta b$. The shell models of different planes are coupled by Alfvén waves propagating along the mean magnetic field [5, 2]. In each layer the damping factor is given (dimensionally) by $\beta=\exp \left[-t_{p h, c} / \tau_{n l}^{p h, c}\right]$, with $\tau_{n l}^{p h, c}=l_{\perp} / u_{p h, c}^{ \pm}$and $l_{\perp}$ is the outer scale of turbulence taken to coincide with the loop width. We expect that the amplitude in the corona saturates to value smaller than $u_{c}^{ \pm} \propto 1 / \varepsilon$ when subject to a damping, say the WKB value $u_{c} \propto 1 / \sqrt{\varepsilon}$. One gets $t_{c} / \tau_{n l}^{c}=\left(u_{1}^{0} \sqrt{\varepsilon} / V_{a}^{p h}\right)\left(L_{c} / l_{\perp}\right)$ that justifies neglecting the damping in the chromosphere. Based on this dimensional analysis, we set $u_{1}^{0}=$

\footnotetext{
2 In their model atmosphere, Grappin et al. [1] have non-vanishing Alfvén speed gradients at the photospheres, corresponding to our T.R..
}

$0.2 \mathrm{~km} / \mathrm{s}, L_{c}=6 \mathrm{Mm} \varepsilon=0.4, L_{c} / l_{\perp}=1, u_{1}^{0} / V_{a}^{p h}=2 / 7$ in the simulation to obtain a weak turbulence limit ${ }^{3}$. The velocity shear between footpoints relaxes in a few characteristic timescales, although a small shear survives, oscillating with time (red-dotted curve in Fig. 3). For smaller $\varepsilon$, relaxation occurs earlier and to larger $\Delta u$ (cf. symbols in Fig. 4, A), with oscillation on similar timescale (not shown). The nature of the oscillations is not completely clear, although it is related to the sharp reflection at the T.R. and is expected to be reduced when the T.R. is properly accounted for.

The asymptotic shear can be understood in our simple analytical picture adopting a phenomenological expression for the turbulent damping. With $\beta<1$ and $\xi=1$ in the recurrence formula, and in the limit of small $\varepsilon$ one obtains for the wave amplitude in the corona:

$$
u_{L}^{+}=\frac{1}{2 \varepsilon} u_{1}^{0} \frac{1-\exp \left[-2 t / \tau_{r e l}-t / \tau_{n l}\right]}{1+\tau_{r e l} / 2 \tau_{n l}} .
$$

At the beginning, when few reflections have occurred, $\tau_{n l} \gg \tau_{r e l}$ and the system evolves as in the linear case, with the wave amplitude in the corona growing toward the asymptotic non-WKB value $u_{L}^{+}=u_{1}^{0} / \varepsilon$. When $\tau_{n l} \lesssim$ $\tau_{r e l}$ the relaxation process stops and the level of fluctuation saturates to the value:

$$
u_{L}^{+}=\frac{1}{2 \varepsilon} u_{1}^{0} \frac{1}{1+\tau_{r e l} / 2 \tau_{n l}}
$$

Assuming that $u_{L}^{+} \approx u_{L}^{-}$(or equivalently $\delta u \ll$ $\delta b_{c} / \sqrt{4 \pi \rho_{c}}$ ), and choosing a Kolmogorov type phenomenology for the nonlinear timescale [6], $\tau_{n l}=l_{\perp} / u_{L}^{+}$, yields the asymptotic level of the coronal magnetic field

$b_{\infty}=\left.\frac{\delta b_{c}^{\infty}}{\sqrt{4 \pi \rho_{c}}} \approx u_{L}^{+}\right|_{\infty}=u_{1}^{0} \frac{\tau_{n l}^{p h}}{\tau_{r e l}}\left[\sqrt{1+\frac{\tau_{r e l}}{\tau_{n l}^{p h}} \frac{1}{\varepsilon}}-1\right]$

Given the value of $u_{c}^{+} \approx u_{c}^{-}$, the velocity shear at footpoints can be obtained from the asymptotic expression or the time evolution formula for $\Delta u$. Here we prefer to exploit the stationary state of the system in the induction equation, which in a dimensional form leads to (other choices gives similar results):

$$
\frac{\partial b_{\infty}}{\partial t} \approx V_{a}^{c} \frac{\Delta u}{L_{c}}-\frac{b_{\infty}}{\tau_{n l}}=0,
$$

from which one finally gets,

$$
\Delta u=\frac{L_{c}}{l_{\perp}} \frac{b_{\infty}^{2}(\varepsilon)}{V_{a}^{p h}} \varepsilon
$$

\footnotetext{
3 Keeping fixed the ratio $u_{1}^{0} / V_{a}^{p h}$, a different choice of the nonlinear timescale, as done below, would give a slightly higher $\varepsilon$.
} 


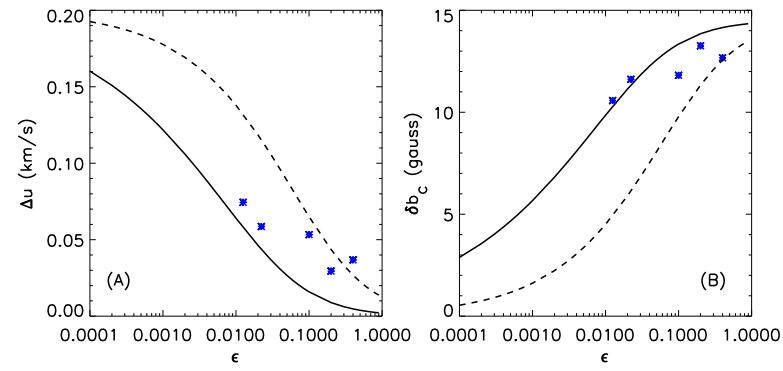

FIGURE 4. Velocity shear $\Delta u$ (A) and asymptotic coronal magnetic field $\delta b_{c}$ (B) as a function of $\varepsilon$ from eq. 12 and eq. 14 solved with different nonlinear timescales: a Kolmogorov (dashed) and an IK (solid) type. Symbols are results from the shell simulations.

With a different choice of the nonlinear timescale, as in the IK phenomenology $[7,8], \tau_{n l}=l_{\perp} V_{a}^{c} /\left(u_{L}^{+}\right)^{2}$, one obtains a cubic expression for $u_{c}^{+}$, which has to be solved numerically, but the induction equation can still be used to get the velocity shear. Note that for small $\varepsilon$, Eq. 13 gives a dependence consistent with the WKB estimate $u_{c}^{ \pm} \propto \varepsilon^{-1 / 2}$, while the IK phenomenology gives $u_{c}^{ \pm} \propto \varepsilon^{-2 / 3}$, that is a coronal magnetic field intermediate between the WKB and linear solutions. In Fig. 4 symbols represent $\delta b_{c}$ and $\Delta u$ computed at the coronal base obtained with the simulations (and averaging over 4-5 oscillations), varying $\varepsilon$ while keeping fixed the other parameters (loop length and width, Alfvén speed at the photosphere, injected wave amplitude). The solid and dashed lines are the analytical estimates obtained with a Kolmogorov and IK phenomenology. A relatively good agreement is found between the simulations at high Alfvén speed contrast (small $\varepsilon$ ) and the IK phenomenology. For the latter a fiarly accurate approximation is given by (cf. Fig. 4, B for $\varepsilon \lesssim 10^{-3}$ ):

$$
\delta b_{c}^{\infty} \approx \delta b_{p h}^{0}\left[\frac{l_{\perp}}{L_{c}}\left(\frac{V_{a}^{p h}}{u_{1}^{0}}\right)^{2} \varepsilon\right]^{1 / 3},
$$

$\left(\delta b_{p h}^{0}=1 / 2 u_{1}^{0} \sqrt{4 \pi \rho_{p h}} \approx 15\right.$ Gauss). For small reflection $(\varepsilon \gtrsim 0.02), u_{c}^{+} \nsim u_{c}^{-}, \delta b_{c} / \sqrt{4 \pi \rho_{c}} \nsim u_{c}^{ \pm}$, so that both phenomenologies fail to reproduce the simulation results.

\section{DISCUSSION}

Adopting a simplified model based on Alfvén wave propagation and reflection we recover the essential features found in Grappin et al. [1]. A loop driven at one footpoint by a "zero-frequency" photospheric velocity relaxes to a uniform transverse velocity $\Delta u=0$ and magnetic field $\delta b_{c}=\delta b_{p h}$ on a characteristic timescale $\tau_{r e l}=L_{c} / V_{a}^{p h}$.
This situation continues to hold basically for $\omega \ll \omega_{*} \approx$ $1 / \tau_{\text {rel }}$. For $\omega \approx \omega_{*}$, the asymptotic shear is finite (partial relaxation) and $\delta b_{c}<\delta b_{p h}$. For $\omega \gg \omega_{*}$, our model correctly reproduces the behavior of the coronal magnetic field that tends to 0 and shows resonances at well defined frequencies. On the countrary oscillations in the velocity shear, $0<\Delta u=u_{1}^{0}$, are exaggerated in our simplified model but when the T.R. is represented as a continuous layer we recover the correct behavior $\Delta u \rightarrow 0$. The system is neither line-tied $(\delta u=0)$ nor relaxed $\left(\delta b_{c}=0\right)$. When weak (turbulent) damping is taken into account, both numerical simulations and analytical calculations show that the relaxation process still occurs. In the strong turbulence limit, the loop relaxation is stopped as soon as the coronal nonlinear time becomes smaller than the coronal propagation time $\left(\tau_{n l}^{c}<t_{c}\right)$. The asymptotic magnetic field in the corona (in velocity units) has a scaling that varies between $\varepsilon^{-1 / 2}$ and $\varepsilon^{-2 / 3}$, depending on the phenomenology (Kolmogorov and IK respectively, the latter showing a better agreement with the simulations), which is intermediate between the linear $(1 / \varepsilon)$ and WKB $(1 / \sqrt{\varepsilon})$ scalings. The relaxation still occurs when the ratio $\tau_{n l}^{c} / \tau_{\text {rel }}>1$. According to the definition of nonlinear timescale in the Kolmogorov or IK phenomenology, this can be rewritten as $\tau_{n l}^{c} / \tau_{r e l}=\left(\varepsilon \tau_{n l}^{p h} / \tau_{r e l}\right)^{1 / 2}$ and $\tau_{n l}^{c} / \tau_{r e l}=\left(\varepsilon \tau_{n l}^{p h} / \tau_{r e l}\right)^{1 / 3}$ respectively. For a given loop width and initial footpoint shear, and assuming that longer loop has higher density contrast (smaller $\varepsilon$ ) the asymptotic state is controlled by loop length.

Acknowledgments This research was carried out in part at the Jet Propulsion Laboratory, California Institute of Technology, under a contract with the National Aeronautics and Space Administration. It was also supported by the Italian Space Agency contract Solar System Exploration and by the Belgian Federal Science Policy Office through the ESA-PRODEX program.

\section{REFERENCES}

1. R. Grappin, G. Aulanier, and R. Pinto, $A \& A$ 490, 353-356 (2008).

2. E. Buchlin, and M. Velli, ApJ 662, 701-714 (2007).

3. F. Malara, G. Nigro, and P. Veltri, "Large-scale energy balance and MHD turbulence in solar coronal structures," in Solar Wind 12 proceedings, 2009.

4. G. Nigro, F. Malara, and P. Veltri, ApJ 685, 606 (2008).

5. G. Nigro, F. Malara, V. Carbone, and P. Veltri, Physical Review Letters 92, 194501-+ (2004).

6. A. N. Kolmogorov, SSSR 30, 299-303 (1941).

7. P. S. Iroshnikov, Soviet Astronomy 7, 566-+ (1964).

8. R. H. Kraichnan, Phys. Fluids 8, 1385-1387 (1964). 